\title{
B-Cell- and Myocyte-Specific E2-Box-Binding Factors Contain E12/E47-Like Subunits
}

\author{
CORNELIS MURRE, ${ }^{1,2}$ ANNA VORONOVA, ${ }^{1}$ AND DAVID BALTIMORE ${ }^{1,3 *}$ \\ Whitehead Institute for Biomedical Research, 9 Cambridge Center, Cambridge, Massachusetts 02142 ${ }^{1}$; \\ Department of Biology, University of California San Diego, La Jolla, California 92093-0116 ${ }^{2}$; and \\ The Rockefeller University, New York, New York $10021^{3}$
}

Received 6 September 1990/Accepted 21 November 1990

\begin{abstract}
Recent studies have identified a family of DNA-binding proteins that share a common DNA-binding and dimerization domain with the potential to form a helix-loop-helix (HLH) structure. Various HLH proteins can form heterodimers that bind to a common DNA sequence, termed the E2-box. We demonstrate here that E2-box-binding B-cell- and myocyte-specific nuclear factors contain subunits which are identical or closely related to ubiquitously expressed (E12/E47) HLH proteins. These biochemical data suggest a pleiotropic function for E12/E47-like molecules in mammalian differentiation, similar to the genetically defined function of daughterless in Drosophila development.
\end{abstract}

One class of DNA-binding proteins contains a region of homology that has the potential to form a helix-loop-helix (HLH) structure preceded by a highly basic region (22); this region has been shown to contain a DNA-binding and dimerization domain $(12,22,29)$. Certain HLH homo- and heterodimers bind to a common DNA sequence, termed the E2-box. The E2-box is present in the immunoglobulin heavyand light-chain gene enhancer, the insulin gene enhancer, and the muscle creatine kinase gene enhancer $(4,7,19,20)$. In each of these enhancers it plays a crucial role in the regulation of tissue-specific gene expression. Representative members of the HLH family that bind the E2-box are E12 (pan-1), E47 (E2-5; pan-2), and E2-2 (16, 22, 24) immunoglobulin enhancer-binding proteins; MyoD (12), myogenin $(3,31)$, and myf-5 (2), three proteins that induce myogenesis; and daughterless (da) $(6,8-11)$ and achaete-scute (28), Drosophila genes that are involved in cell determination and differentiation. In vitro evidence implies that classes of HLH proteins use combinatorial interactions to control tissuespecific gene expression in various developmental pathways (21). Class A includes ubiquitously expressed proteins $d a$, E12, E2-2, and E47. E12 and E47 are differentially spliced products of the same gene (26b) and share most of their amino acid sequence. All four proteins have very closely related HLH motifs. Class B includes tissue-specific proteins, MyoD, myogenin, myf-5, and members of the achaetescute gene family. Class B proteins bind in vitro with high affinity to the E2-box as heterodimers with class A proteins. In addition, there is a class of HLH proteins which lack a basic region and thus are negative regulators of DNA binding (Id, extramacrochaete) $(1,14,15)$. To examine whether HLH heterodimers are also formed in vitro, E2-box-binding proteins from a variety of cell lines were characterized. We show here that B-cell- and muscle-specific E2-box-binding factors contain components that are identical or antigenically related to the E12/E47 proteins.

\section{MATERIALS AND METHODS}

Cell lines. HeLa and LA-N-5 (26) cells were grown in Dulbecco modified Eagle medium supplemented with $5 \%$

* Corresponding author. (vol/vol) fetal calf serum. PD31 and WEHI 231 cells were grown in RPMI medium containing $10 \%$ fetal calf serum. $\mathrm{Ba} / \mathrm{F}$ and LyD9 were grown in RPMI medium containing $20 \%$ fetal calf serum and interleukin-3 (27).

Plasmids. A 2-kb cDNA fragment containing the entire coding region of $\mathrm{E} 47$ was inserted into the $\mathrm{XbaI}$ site of the pBluescript II $\mathrm{SK}^{-}$vector (Stratagene) (28a) for in vitro transcription with T3 RNA polymerase. A CDM8 vector containing the 2-kb E47 cDNA insert at its BstXI site was used for expression in eucaryotic cells (25).

Extract preparation and gel shift assays. Nuclear extracts were prepared exactly as described by Dignam et al. (13). Oligonucleotides containing the wild-type monomeric $\mu \mathrm{E} 2$ and muscle creatine kinase E2-box sequence were ${ }^{32} \mathrm{P}$ labeled $(50,000 \mathrm{cpm} / \mathrm{ng})$, and $0.5 \mathrm{ng}$ of DNA probe was incubated with 3 to $10 \mu \mathrm{g}$ of nuclear extract. The $\mathrm{kE2}$ probe was derived from a restriction fragment as described previously (22). The binding reaction mixtures were incubated at room temperature for $15 \mathrm{~min}$ and contained $10 \mathrm{mM}$ Tris $\mathrm{pH}$ 7.5), $50 \mathrm{mM} \mathrm{NaCl}, 1 \mathrm{mM}$ dithiothreitol, $1 \mathrm{mM}$ EDTA, $5 \%$ glycerol, and 0.5 or $1 \mu \mathrm{g}$ of poly(dIC-dIC) as the nonspecific competitor. The complexes were resolved by electrophoresis through $4 \%$ polyacrylamide gels containing $50 \mathrm{mM}$ Tris, $0.38 \mathrm{M}$ glycine, and $2 \mathrm{mM}$ EDTA. For competition experiments the conditions were exactly as described above, except that specific and nonspecific competitor DNAs were included in the mixture (in the amounts detailed in the figure legends) prior to the addition of protein. For supershift assays, nuclear extracts were incubated for $15 \mathrm{~min}$ with antibody prior to incubation with labeled DNA.

In vitro transcription and translation. RNA was synthesized in a $100-\mu l$ reaction for $2 \mathrm{~h}$ at $40^{\circ} \mathrm{C}$ with a substrate of $2 \mu \mathrm{g}$ of linearized DNA and with T3 or T7 RNA polymerase (Stratagene) in the presence of RNasin (Boehringer Mannheim). The RNA was phenol and chloroform extracted once and ethanol precipitated. The ethanol precipitate was dried and resuspended in $20 \mu \mathrm{l}$ of water. In vitro translation of the RNAs was done by using a rabbit reticulocyte lysate (Promega) in a 50- $\mu$ l reaction with $10 \%$ of the synthesized RNA as a substrate. Proteins were synthesized at $30^{\circ} \mathrm{C}$ for 1 h. Reaction conditions were as recommended by the manufacturer (Promega). 


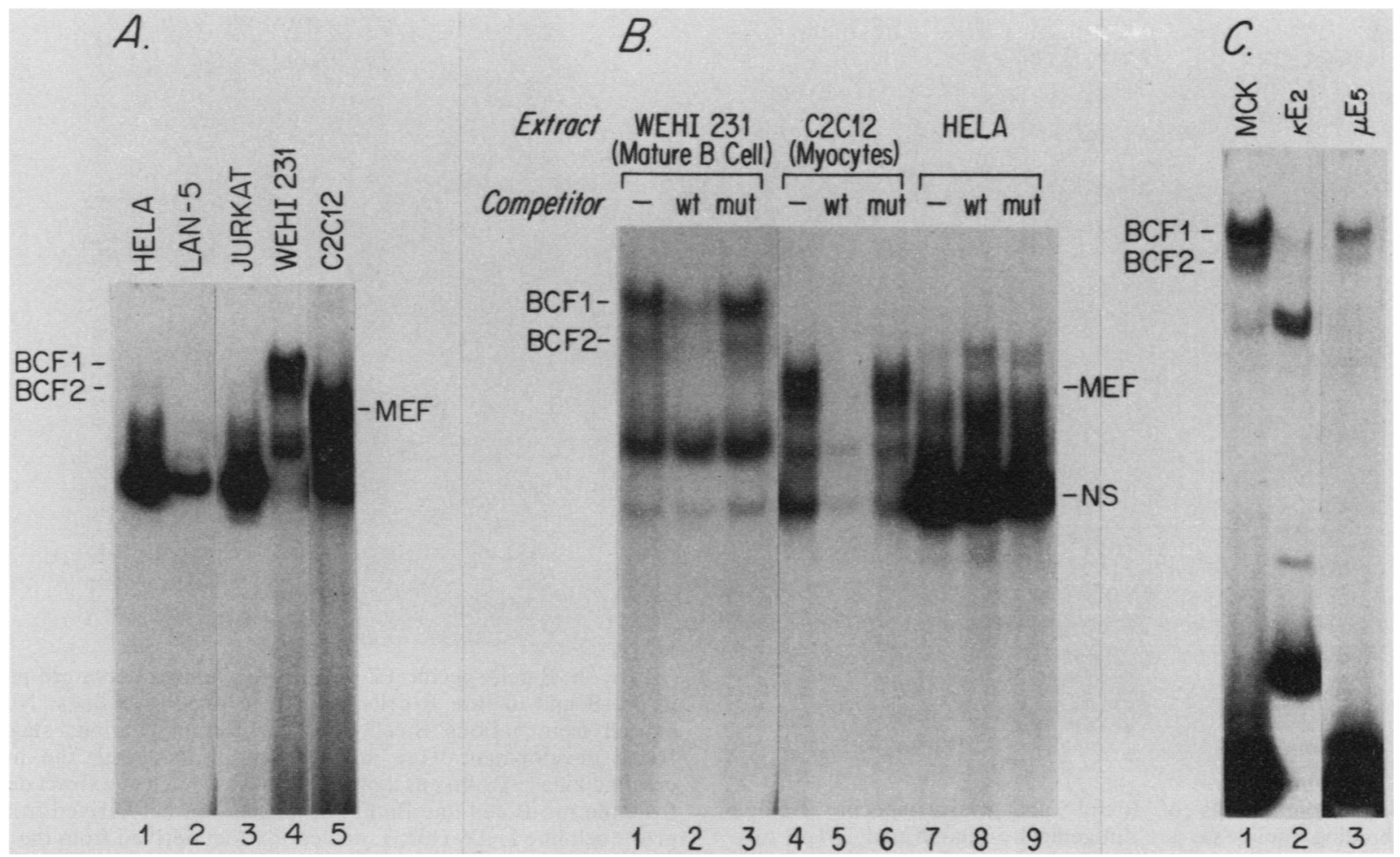

FIG. 1. Binding of proteins to the E2-box motif. (A) B-cell and myocyte nuclear extracts contain tissue-specific muscle creatine kinase enhancer E2-box DNA-binding proteins. Lanes: 1, HeLa extract; 2, LA-N-5 extract (neuroblastoma); 3, Jurkat extract (T cell); 4, WEHI 231 extract (mature B cell); 5, C2C12 extract (muscle cell). (B) B-cell- and myocyte-specific proteins bind specifically to the muscle creatine kinase enhancer E2-box. Lanes: 1, WEHI 231 extract with no competitor added (-); 2, WEHI 231 extract in the presence of 10 ng of unlabeled wild-type (wt) E2-box competitor DNA; 3, WEHI 231 extract in the presence of 10 ng of unlabeled mutant (mut) E2-box competitor DNA; 4, C2C12 extract with no competitor DNA added; 5, C2C12 extract in the presence of $10 \mathrm{ng}$ of unlabeled E2-box competitor DNA; 6, C2C12 extract with 10 ng of unlabeled mutant E2-box competitor DNA; 7, HeLa extract with no competitor DNA added; 8, HeLa extract with 10 ng of unlabeled E2-box competitor DNA added; 9, HeLa extract with $10 \mathrm{ng}$ of unlabeled mutant competitor DNA added. (C) B-cell-specific DNA-binding proteins bind to the E2-box present in the muscle creatine kinase enhancer and to $\mu E 5$ and $\kappa E 2$ sequences present in the immunoglobulin enhancers. Lanes: 1, WEHI 231 extract analyzed by using the E2-box present in the muscle creatine kinase enhancer as a probe; 2, WEHI 231 extract analyzed by using the E2-box present in the $\mathrm{kE2}$ site of the kappa light-chain enhancer as a probe; 3, WEHI 231 extract analyzed by using the E2-box present in the $\mu E 5$ site of the immunoglobulin heavy-chain enhancer as a probe. MEF, Muscle-specific complexes; NS, nonspecific DNA-binding proteins.

\section{RESULTS}

B-cell-specific E2-box DNA-binding proteins. B-cell-specific proteins binding to the E-box sites in the immunoglobulin heavy-chain enhancer were first detected by in vivo methylation protection experiments (7). Previous studies with low-ionic-strength gel systems failed to detect such B-cell-specific E2-box-binding proteins in vitro $(17,19)$. Recently, tissue-specific E2-box-binding proteins were found in muscle-derived nuclear extracts $(3,4,18)$. To look for other tissue-specific E2-box-binding proteins, we screened a wide variety of nuclear extracts by using an electrophoretic mobility shift assay (EMSA) at high ionic strength. As demonstrated before $(3,4,18)$, muscle-specific proteins, designated as MEF-1, are present in myocyte nuclear extracts and bind the E2-box sequence present in the muscle creatine kinase enhancer (Fig. 1A, lane 5). Nuclear extracts derived from HeLa cells (lane 1), the T-cell line Jurkat (23) (lane 3), and the neuroblastoma cell line LA-N-5 (26) (lane 2) did not contain complexes that migrated in a cell-type-specific manner. However, nuclear extracts derived from a mature B-cell line, WEHI 231, contained two B-cell-specific complexes, termed BCF1 and BCF2, which were not found in extracts of the other tested cell types (lane 4). Thus, in addition to myocytes, distinct but tissue-specific E2-box-binding proteins are present in nuclear extracts derived from $B$ cells.

To determine whether the B-cell- and myocyte-specific complexes interact with the E2-box in a specific manner, we performed a competition analysis. DNA containing the wildtype E2-box sequence, derived from the muscle creatine kinase enhancer (GCAGGTGT), did compete for BCF1 and BCF2 binding (Fig. 1B, lane 2), whereas DNA containing a mutant E2-box sequence (TACCGTGT) did not compete (lane 3). Similarly, DNA containing the wild-type E2 sequence competed for binding to muscle-specific complexes (18) (Fig. 1B, lane 5), whereas DNA containing the mutant E21 sequence did not compete (18) (lane 6).

To test whether BCF1 and BCF2 can interact with the E2-box sequences present in the heavy-chain and kappa light-chain enhancer, nuclear extracts from WEHI 231 were incubated with probes containing the $\kappa E 2$ (GGCAGGTGG) (22) and $\mu$ E5 (TGCAGGTGT) sites. Both $\kappa E 2$ and $\mu E 5$ are crucial for immunoglobulin heavy- and light-chain gene expression (19). BCF complexes bind to $\mu \mathrm{E} 5$ (Fig. 1C, lane 


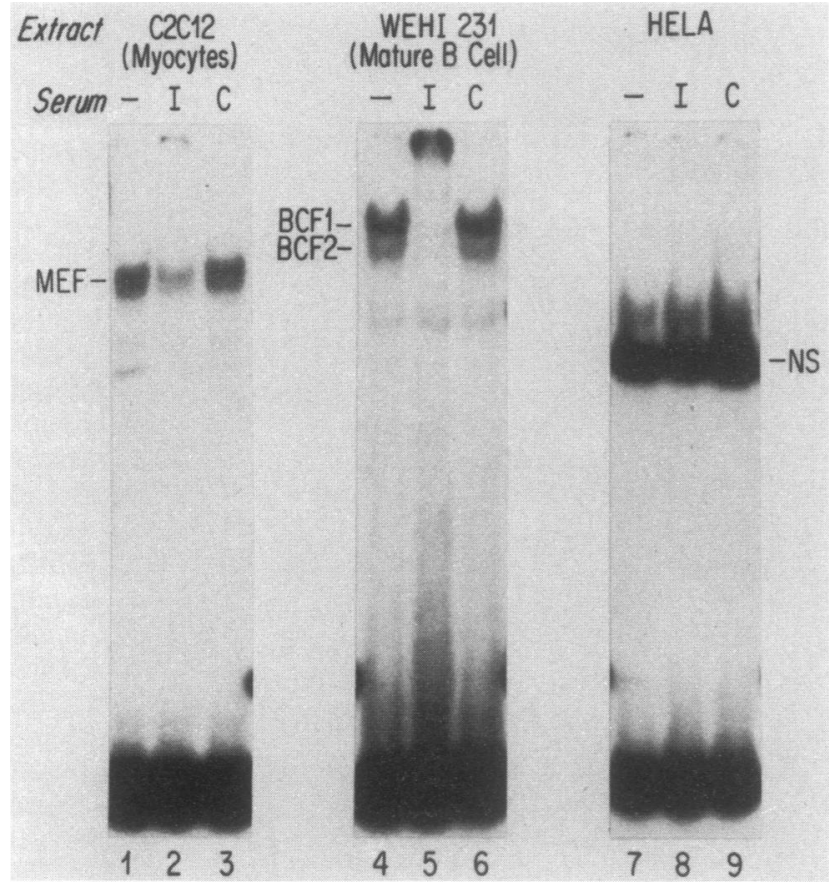

FIG. 2. Components of B-cell- and muscle-specific E2-box DNA-binding complexes are antigenically related to E12/E47 proteins. Lanes: 1, muscle ( $\mathrm{C} 2 \mathrm{C} 12)$ nuclear extracts incubated in the absence of antiserum; 2 , muscle extract incubated in the presence of anti-E12/E47 antiserum; 3, muscle extract incubated in the presence of preimmune antiserum; 4, B-cell extract incubated in the absence of antiserum; 5, B-cell extract incubated in the presence of anti-E12/ E47 antiserum; 6, B-cell extract incubated in the presence of preimmune serum; 7, HeLa extract incubated in the absence of antiserum; 8, HeLa extract incubated in the presence of anti-E12/ E47 serum; 9, HeLa extract incubated in the presence of preimmune serum. MEF, Muscle-specific complexes; NS, nonspecific DNAbinding proteins.

3) with high affinity, but binding to the $\kappa E 2$ site was weaker and qualitatively different (lane 2). The faster-migrating complex binding to the $\mathrm{kE2}$ site was nonspecific. Thus, BCF complexes bind to sequences that are important for immunoglobulin heavy- and light-chain gene expression $(17,19)$.

B-cell-specific E2-box DNA-binding complexes contain E12/ E47-like subunits. E47 protein can transactivate a reporter gene containing multiple $\mu \mathrm{E} 5$ and $\kappa \mathrm{E} 2$ sequences positioned $5^{\prime}$ of its promoter $(16,29 a)$, suggesting that E47 is part of a transcriptional complex binding to the $\mu \mathrm{E} 5$ and $\kappa E 2$ motifs. To test whether the BCF complexes contain E12/E47 molecules, B-cell nuclear extracts derived from WEHI 231 were incubated with anti-E12/E47 antiserum and assayed by EMSA. The antiserum does not recognize determinants present in the conserved HLH motif or determinants present in other related $\mathrm{HLH}$ proteins, for example MyoD, Id, and myogenin among the tested molecules $(1,21,31)$. Thus, it is specific for E12 and E47 polypeptides, although we cannot exclude the possibility that other closely related polypeptides share determinants with E12/E47. The anti-E12/E47 antibody, however, does not distinguish between E12 and E47 polypeptides (21). Both the BCF1 and BCF2 complexes supershifted (migrated more slowly) in the presence of the anti-E12/E47 antibody (Fig. 2, lane 5), whereas preimmune serum did not affect complex mobility (lane 6). Thus, BCF1 and BCF2 contain E47- and/or E12-like molecules.

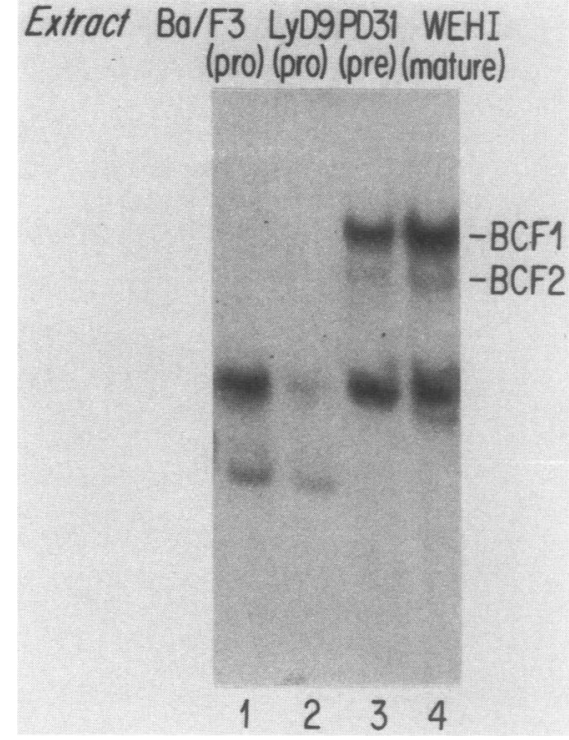

FIG. 3. B-cell-specific E2-box DNA-binding factors are present in pre-B and mature B cells but not in pro-B cell lines. Nuclear extract from various B-cell lines representing various stages of B-cell development were analyzed by EMSA with the muscle creatine kinase E2-box as a probe. Lanes: 1, nuclear extract derived from the pro-B cell line $\mathrm{Ba} / \mathrm{F} 3 ; 2$, nuclear extract derived from the pro-B cell line LyD9 (13); 3, nuclear extract derived from the pre-B cell line PD31; 4, nuclear extract derived from the mature B-cell line WEHI 231. Only the region of the gel containing the shifted probe is shown.

BCF1 and BCF2 are present in pre-B and mature $B$ cells but not in pro-B cells. To determine at what stages of B-cell development BCF1 and BCF2 are present, nuclear extracts derived from different B-cell lines were examined by EMSA with the E2-box, derived from the muscle creatine kinase enhancer, as a probe. Both BCF1 and BCF2 were detected in pre-B-cell and mature B-cell lines (Fig. 3, lanes 3 and 4), but not in pro-B-cell lines (a kind gift of R. Palacios) (lanes 1 and 2) (27). We have examined four additional pre-B-cell and mature B-cell lines; they all contain the B-cell-specific BCF1 and BCF2 complexes (data not shown). The faster-migrating band present in pro-B cells is nonspecific (20a). Mature $B$ and pre-B-cell lines, but not the pro-B-cell lines examined here, express immunoglobulin heavy chain. The presence of BCF1 and BCF2 therefore correlates with immunoglobulin heavy-chain gene expression.

Myocyte-specific E2-box DNA-binding proteins contain E12/ E47-like subunits. Previously we have shown that E12 and E47 proteins can interact with $\mathrm{MyoD}$ in vitro to form heterodimers that bind to the E2-box motif (21). Myocyte nuclear extracts contain muscle-specific complexes that are identical or antigenically related to $\mathrm{MyoD}$ and myogenin (18). To determine whether E12/E47-like proteins form heterodimers with MyoD and myogenin in vivo, myocyte nuclear extracts were incubated with an anti-E12/E47 antibody and analyzed by EMSA. The muscle-specific complexes reacted with the antibody (Fig. 2, lane 2) but not with preimmune serum (lane 3 ). Thus, myocyte-specific complexes contain E12/E47 molecules or ones closely related to E12/E47.

BCF complexes comigrate with homo-oligomers of E47 in an EMSA. Although the anti-E12/E47 antibody did react with 


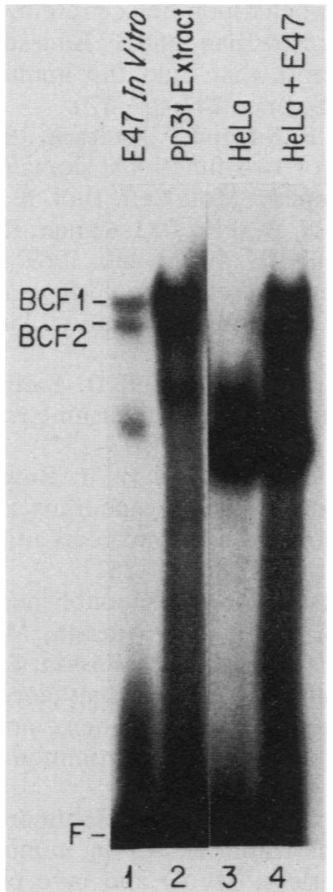

FIG. 4. $B C F 1$ and $B C F 2$ proteins comigrate in an EMSA with homo-oligomers of E47. BCF1 and BCF2 were analyzed by EMSA and compared in vitro and in vivo by using a radiolabeled muscle creatine kinase E2-box as a probe. Lanes: 1, in vitro-translated E47; 2, nuclear extract derived from a pre-B cell line, PD31; 3, nuclear extract derived from HeLa cells; 4, nuclear extract derived from HeLa cells transiently transfected with an E47 cDNA placed under the control of the cytomegalovirus promoter. F, Probe not bound to protein.

the muscle-specific complexes, the relative ability of the antiserum to supershift the complexes was 100 -fold lower for the muscle-specific complexes than for the BCF proteins (20a). A trivial explanation for this difference in affinity could be that $\mathrm{BCF} 1$ and $\mathrm{BCF} 2$ are homo-oligomers of E12/E47. To address this issue, we have examined the relative affinity of the anti-E12/E47 antibody for in vitro-synthesized E47 homo-oligomers and E47-MyoD heterodimers. E47 homooligomers have a 100 -fold-higher affinity for the antibody than do E47-MyoD heterodimers (20a). In addition, in vitrotranslated full-length E47 generated two complexes that comigrated with the $\mathrm{BCF} 1$ and $\mathrm{BCF} 2$ complexes present in nuclear extracts from a pre-B-cell line, PD31 (Fig. 4, lanes 1 and 2). Why the in vitro E47-synthesized molecules generate two complexes with different mobilities in an EMSA is not clear. They either could represent variable complexes of $\mathrm{E} 47$, for example a dimer (BCF2) and a tetramer (BCF1), or could result from posttranslational modification. To determine whether the product of E47 cDNA migrates with similar mobility when expressed in vivo, a full-length E47 cDNA placed under the control of the cytomegalovirus promoter was introduced transiently into HeLa cells. Nuclear extracts derived from HeLa cells transfected with E47 cDNA were then examined by EMSA. Two complexes were apparent in the extracts prepared from the transfected cells but not from the control cells. These complexes comigrated exactly with BCF1 and BCF2 (Fig. 4, lanes 3 and 4) and were supershifted by the anti-E12/E47 antibody (data not shown). These data suggest that the BCF proteins might consist of oligomers of E47 or E12 or a closely related protein. In addition, in vitro-translated E47 polypeptide and BCF proteins have very similar affinities to anti-E12/E47 antibody (20a), which further supports the idea that the BCF proteins are indeed oligomers of E12 or E47. It is unlikely that BCF1 and BCF2 represent homo-oligomers of E12, because E12 homo-oligomers have a relatively weak affinity for the E2 sequence under the conditions described here (22). Thus, BCF1 and BCF2 most probably represent homo-oligomers of E47 or hetero-oligomers containing E12 and E47. We note, however, that further analysis of these complexes is required to exclude the possibility that a B-cell-specific polypeptide associates with E47 or E12 in BCF1 and BCF2.

\section{DISCUSSION}

We have presented data showing that both myocyte and B-cell E2-box DNA-binding proteins contain E12/E47-like subunits. In B-cell nuclear extracts, complexes containing E47 subunits-very probably consisting of a homo-oligomer of E47-are present that cannot be detected in non-B cells. E12 and E47 mRNA has been detected in all cell types examined (22). We have immunoprecipitated E12/E47 polypeptides from Jurkat and HeLa cells $(20 \mathrm{a})$. Why are apparent E47 homo-oligomers detected in nuclear extracts derived from B cells but not in those of other cell types? One possibility is that in many cells E47 polypeptides are complexed with other HLH polypeptides, such as Id, that would inhibit their DNA binding (1). Id was shown to form heterodimers with E47 in vitro, inactivating its ability to bind to DNA. Id levels are low in differentiated cell types, whereas they are high in undifferentiated cells (1). High levels of Id might inhibit the DNA-binding activity or HLH proteins such as E47. In pro-B and other cell types, levels of inhibitory molecules identical or related to Id might be high, preventing E47 from forming homo-oligomers (24a). During B-cell differentiation, similarly to myogenesis, levels of such inhibitory molecules might drop, allowing E47 polypeptides to form oligomers that can now bind to regulatory sequences present in the immunoglobulin heavy- and light-chain gene enhancers, inducing their transcription and subsequently B-cell differentiation. In other cells, the presence of class B HLH proteins such as MyoD would lead to preferential heterodimer formation (26b) and suppress the homo-oligomers:

These data suggest that the role of E12/E47-like proteins in mammalian development might be similar to that of $d a$ in Drosophila. da is a gene whose various mutants show pleiotropic effects. $d a$ controls both sex determination and neurogenesis (9). A role for $d a$ in other developmental pathways is likely, but evidence is lacking because of the lethality of $d a$ mutations. The physical similarity between $d a$ and E12/E47 proteins is striking. The E12/E47 sequences are as related to $d a$ in the region surrounding the HLH region as they are to each other (22). In addition, each of the proteins is ubiquitously expressed. In this paper we present data suggesting a role for E12/E47-like proteins in myogenesis and B-cell development. A role for E12/E47 proteins in pancreatic $\beta$-cell differentiation is likely as well. The E2-box sequence is present in the insulin enhancer, a site that has been shown to be crucial for its tissue-specific expression $(20,24)$. Indeed, Stein and collaborators recently found that a $\beta$-cell-specific complex that binds the E2-box is supershifted with an anti-E12/E47 antibody (26a, 30). They also found that the activity of the $\mathrm{E} 2$ regulatory sequence present in the insulin enhancer is responsive to Id. Thus, E12/E47 
and related molecules may well function in controlling development by associating either with tissue-specific components, such as MyoD in MEF-1, or as homo-oligomers in the BCF proteins, inducing the differentiated-muscle-, B-cell-, and $\beta$-cell-specific phenotypes.

\section{ACKNOWLEDGMENTS}

We thank Astar Winoto, Stephen Smale, and Patrick Matthias for generously providing nuclear extracts and Helen Blau for $\mathrm{C} 2 \mathrm{C} 12$ cells.

C.M. was supported by a fellowship from the Leukemia Society of America. A.V. was supported by a fellowship from the American Cancer Society. This work was supported by Public Health Service grant GM39458.

\section{REFERENCES}

1. Benezra, R., R. L. Davis, D. Lockhon, D. L. Turner, and H. Weintraub. 1990. The protein Id: a negative regulator of helixloop-helix DNA binding proteins. Cell 61:49-59.

2. Braun, T., G. Buschhausen-Denker, E. Bober, E. Tannich, and H. H. Arnold. 1989. A novel human muscle factor related to but distinct from MyoD1 induces myogenic conversion in 10T1/2 fibroblasts. EMBO J. 8:701-709.

3. Brennan, T. J., and E. N. Olson. 1990. Myogenin resides in the nucleus and aquires high affinity for a conserved enhancer element on heterodimerization. Genes Dev. 4:582-595.

4. Buskin, J. N., and S. D. Hauschka. 1989. Identification for a myocyte nuclear factor which binds to the muscle-specific enhancer of the mouse muscle creatine kinase gene. Mol. Cell. Biol. 9:2627-2640.

5. Caudy, M., E. H. Grell, C. Dambly-Chaudiere, A. Ghysen, L. Y. Jan, and Y. N. Jan. 1988. The maternal sex determination gene daughterless has zygotic activity necessary for the formation of peripheral neurons in Drosophila. Genes Dev. 2:843-852.

6. Caudy, M., H. Vaessin, M. Brand, R. Tuma, L. Y. Jan, and Y. N. Jan. 1988. Daughterless, a gene essential for both neurogenesis and sex determination in Drosophila, has sequence similarities to myc and the achaete-scute complex. Cell 55:10611067.

7. Church, G. M., A. Ephrussi, W. Gilbert, and S. Tonegawa. 1985. Cell type specific contacts to immunoglobulin enhancers in nuclei. Nature (London) 313:798-801.

8. Cline, T. W. 1976. A sex-specific, temperature-sensitive maternal effect of the daughterless mutation of Drosophila melanogaster. Genetics 84:723-742.

9. Cline, T. W. 1989. The affairs of daughterless and the promiscuity of developmental regulators. Cell 59:231-234.

10. Cronmiller, C., and T. W. Cline. 1987. The sex determination gene daughterless has different functions in the germ line versus the soma. Cell 48:479-487.

11. Cronmiller, C., P. Schedl, and T. W. Cline. 1988. Molecular characterization of daughterless, a Drosophila sex determination gene with multiple roles in development. Genes Dev. 2:1666-1676.

12. Davis, R. L., P. F. Cheng, A. B. Lassar, and H. Weintraub. 1990. The MyoD DNA binding domain contains a recognition code for muscle-specific gene activation. Cell 60:773-746.

13. Dignam, J. D., R. M. Lebovitz, and R. G. Roeder. 1983. Accurate transcription initiation by RNA polymerase II in a soluble extract from isolated mammalian nuclei. Nucleic Acids Res. 11:1475-1489.

14. Ellis, H. M., D. R. Spann, and J. W. Posakony. 1990. Extramacrochaete, a negative regulator of sensory organ development in Drosophila, defines a new class of helix-loop-helix proteins. Cell 61:27-38.

15. Garrell, J., and J. Modolel. 1990. The Drosophila extramacrochaete locus, an antagonist of proneural genes that like these genes, encodes a helix-loop-helix protein. Cell 61:39-48.

16. Henthorn, P., M. Kiledjian, and T. Kadesch. 1990. Two distinct transcription factors that bind the immunoglobulin enhancer $\mu E 5 / \kappa E 1$ motif. Science 247:467-470.

17. Kiledjian, M., L. K. Su, and T. Kadesch. 1988. Identification and characterization of two functional domains within the murine heavy-chain enhancer. Mol. Cell. Biol. 8:145-149.

18. Lassar, A. B., J. N. Buskin, D. Lockhon, R. L. Davis, S. Apone, S. D. Hauschka, and H. Weintraub. 1989. MyoD is a sequencespecific DNA binding protein, requiring a region of myc homology to bind to the muscle creatine kinase enhancer. Cell 58:823-831.

19. Lenardo, M., J. W. Pierce, and D. Baltimore. 1987. Proteinbinding sites in Ig enhancers determine transcriptional activity and inducibility. Science 236:1573-1577.

20. Moss, L. G., J. B. Moss, and W. J. Rutter. 1988. Systematic binding analysis of the insulin gene transcription control region: insulin and immunoglobulin enhancers utilize similar transactivators. Mol. Cell. Biol. 8:2620-2627.

20a.Murre, C., and D. Baltimore. Unpublished data.

21. Murre, C., P. S. McCaw, H. Vaessin, M. Caudy, L. Y. Jan, Y. N. Jan, C. V. Cabrera, J. N. Buskin, S. D. Hauschka, A. B. Lassar, H. Weintraub, and D. Baltimore. 1989. Interactions between heterologous helix-loop-helix proteins generate complexes that bind specifically to a common DNA sequence. Cell 58:537-544.

22. Murre, C., P. S. McCaw, and D. Baltimore. 1989. A new DNA binding and dimerization motif in immunoglobulin enhancer binding, daughterless, MyoD and myc proteins. Cell 56:777783.

23. Nagasawa, K., A. Howatsan, and T. Mak. 1981. Induction of human $\mathrm{T}$ lymphoblastoid cell lines, Molt-3 and Jurkat by 12-0tetra-decanoyl-phorbol-13 acetate. J. Cell. Physiol. 109:181187.

24. Nelson, C., L. P. Shen, A. Meister, E. Fodor, and W. J. Rutter. 1990. Pan: a transcriptional regulator that binds chymotrypsin, insulin and AP-4 enhancer motifs. Genes Dev. 4:1035-1044.

24a.Schlissel, M., and D. Baltimore. Unpublished data.

25. Seed, B. 1987. An LFA-3 cDNA encodes a phospholipid-linked membrane protein homologous to its receptor CD2. Nature (London) 329:840-842.

26. Seeger, R. C., S. A. Rayner, A. Banerjee, H. Chung, W. E. Laug, H. B. Neustein, and W. F. Benedict. 1977. Morphology, growth, chromosomal pattern and fibrinolytic activity of two new human neuroblastoma cell lines. Cancer Res. 37:1364-1371.

26a.Stein, R. Personal communication.

26b.Sun, $X$., and D. Baltimore. Cell, in press.

27. Takeda, S., S. Gillis, and R. L. Palacios. 1989. In vitro effects of recombinant interleukin 7 on growth and differentiation of bone marrow pro-B and pro-T lymphocyte clones and fetal thymocyte clones. Proc. Natl. Acad. Sci. USA 86:1634-1638.

28. Villares, R., and C. V. Cabrera. 1987. The achaete-scute gene complex of D. melanogaster: conserved domains in a subset of genes required for neurogenesis and their homology to myc. Cell 50:415-424.

28a. Voronova, A. Unpublished data.

29. Voronova, A., and D. Baltimore. 1990. Mutations that disrupt DNA binding and dimer formation in the E47 HLH protein map to distinct domains. Proc. Natl. Acad. Sci. USA 87:4722-4726.

29a.Voronova, A., and D. Baltimore. Unpublished data.

30. Whelan, J., S. R. Cordle, E. Henderson, P. A. Weil, and R. Stein. 1990. Identification of a pancreatic $\beta$-cell insulin gene transcription factor that binds to and appears to activate celltype-specific gene expression: its possible relationship to other cellular factors that bind to a common insulin gene sequence. Mol. Cell. Biol. 10:1564-1572.

31. Wright, W. E., D. A. Sassoon, and V. K. Lin. 1989. Myogenin, a factor regulating myogenesis, has a domain homologous to MyoD. Cell 4:607-617. 